\title{
Laparoscopic versus open splenectomy for nontraumatic diseases
}

\author{
Maurus, C F ; Schäfer, M ; Müller, M K ; Clavien, P A ; Weber, M
}

\begin{abstract}
BACKGROUND: Laparoscopic splenectomy (LS) is the standard procedure for normal size or moderately enlarged spleens; open splenectomy (OS) is preferred in cases of splenomegaly. In this study, indications for and complications of open and laparoscopic splenectomy were analyzed, with the aim to identify patients who will benefit from either technique. METHOD: A consecutive series of 52 patients undergoing elective open or laparoscopic splenectomy between January 2001 and December 2006 was analyzed. Spleen volume was calculated as length $\mathrm{x}$ width $\mathrm{x}$ depth from the pathologist's measurements. RESULTS: LS was performed in 25 patients with a median age of 41 years (range $=24-65$ ). OS was performed in 27 patients with a median age of 60 years (range $=24-86)(\mathrm{p}<0.001)$. Conversion to OS was necessary in two patients $(8 \%)$. Operation time was significantly shorter in LS $(\mathrm{p}<0.05)$. Spleen volume was significantly greater in patients who underwent open (median $=2520$ ml, range $=$ $150-16,800 \mathrm{ml})$ versus laparoscopic $($ median $=648 \mathrm{ml}$, range $=150-4860 \mathrm{ml})$ splenectomy $(\mathrm{p}=0.001)$. In $36 \%$ of all laparoscopic procedures, spleen volume exceeded $1000 \mathrm{ml}$. The underlying disease was mainly immunothrombocytopenia in LS patients and lymphoma and osteomyelofibrosis in OS patients. Five patients died after OS. Significantly more patients were hospitalized longer than 7 days following OS than following LS $(\mathrm{p}<0.05)$. Overall complication rate was higher after OS (LS, 8; OS, 13 patients; $\mathrm{p}<0.05)$. CONCLUSIONS: LS was preferred in younger patients with moderate splenomegaly, while massive splenomegaly mostly led to OS. In view of the absence of technique-related differences, LS can primarily be attempted in all patients.
\end{abstract}

DOI: https://doi.org/10.1007/s00268-008-9727-1

Posted at the Zurich Open Repository and Archive, University of Zurich

ZORA URL: https://doi.org/10.5167/uzh-8308

Journal Article

Published Version

Originally published at:

Maurus, C F; Schäfer, M; Müller, M K; Clavien, P A; Weber, M (2008). Laparoscopic versus open splenectomy for nontraumatic diseases. World Journal of Surgery, 32(11):2444-2449.

DOI: https://doi.org/10.1007/s00268-008-9727-1 


\title{
Laparoscopic Versus Open Splenectomy for Nontraumatic Diseases
}

\author{
Christine F. Maurus • Markus Schäfer • \\ Markus K. Müller · Pierre-Alain Clavien · \\ Markus Weber
}

Published online: 2 September 2008

(C) Société Internationale de Chirurgie 2008

\begin{abstract}
Background Laparoscopic splenectomy (LS) is the standard procedure for normal size or moderately enlarged spleens; open splenectomy (OS) is preferred in cases of splenomegaly. In this study, indications for and complications of open and laparoscopic splenectomy were analyzed, with the aim to identify patients who will benefit from either technique.

Method A consecutive series of 52 patients undergoing elective open or laparoscopic splenectomy between January 2001 and December 2006 was analyzed. Spleen volume was calculated as length $\times$ width $\times$ depth from the pathologist's measurements.

Results LS was performed in 25 patients with a median age of 41 years (range $=24-65$ ). OS was performed in 27 patients with a median age of 60 years (range $=24-86$ ) $(p<0.001)$. Conversion to OS was necessary in two patients $(8 \%)$. Operation time was significantly shorter in LS $(p<0.05)$. Spleen volume was significantly greater in patients who underwent open (median $=2520 \mathrm{ml}$, range $=$ $150-16,800 \mathrm{ml}$ ) versus laparoscopic (median $=648 \mathrm{ml}$, range $=150-4860 \mathrm{ml})$ splenectomy $(p=0.001)$. In $36 \%$ of all laparoscopic procedures, spleen volume exceeded $1000 \mathrm{ml}$. The underlying disease was mainly immunothrombocytopenia in LS patients and lymphoma and osteomyelofibrosis in OS patients. Five patients died after OS. Significantly more patients were hospitalized longer than 7 days following OS than following LS $(p<0.05)$.
\end{abstract}

C. F. Maurus · M. Schäfer · M. K. Müller · P.-A. Clavien · M. Weber $(\bowtie)$

Department of Visceral and Transplantation Surgery, University Hospital Zürich, Rämistrasse 100, 8091 Zürich, Switzerland

e-mail: markus.weber@usz.ch
Overall complication rate was higher after OS (LS, 8; OS, 13 patients; $p<0.05$ ).

Conclusions LS was preferred in younger patients with moderate splenomegaly, while massive splenomegaly mostly led to OS. In view of the absence of techniquerelated differences, LS can primarily be attempted in all patients.

\section{Introduction}

Laparoscopic splenectomy (LS) rapidly gained widespread acceptance for the treatment of nontraumatic diseases of the spleen during the last 10 years. Increased technical skills and technical developments have extended the indications for the laparoscopic removal of the spleen [1]. General indications for a total splenectomy are hemolytic anemias, purpuras, secondary hypersplenism, and some other primary hematologic diseases [2]. Hematologic diseases such as idiopathic thrombocytopenic purpura (ITP) and thrombotic thrombocytopenic purpura (TTP) with normal or only moderately enlarged spleens are still the most common indications for a laparoscopic splenectomy [3]. Malignancy is not a contraindication for a laparoscopic approach since it has recently been demonstrated that morbidity and mortality of patients who underwent laparoscopic splenectomy for benign or malignant diseases were not different and the long-term survival was not impaired [4].

Massively enlarged spleens are often difficult to handle intraoperatively, and there is concern about higher conversion rates and higher morbidity [5]. Retrieving a large specimen may require a long incision so that the benefits of minimally invasive access of LS are wasted [5]. However, the spleen can be morcellated and removed in a bag, with 
minimal risk of spleen cell spreading. Hence, patients with a bulky spleen revealing a long axis of more than $20 \mathrm{~cm}$ are commonly excluded from a laparoscopic approach [6]. Other authors advocate open splenectomy (OS) in patients with a spleen larger than $1 \mathrm{~kg}$ or a splenic axis greater than $15 \mathrm{~cm}$ [7]. In contrast, it has been suggested that with increasing experience, massively enlarged spleens can also be safely removed by a laparoscopic approach [8].

Several studies from centers dedicated to laparoscopy have shown the well-known advantages of minimally invasive surgery, e.g., decreased postoperative pain, shorter hospital stay, and reduced recovery time [9]. A recently published meta-analysis revealed a significantly lower complication rate after laparoscopic versus open splenectomy; in particular, pulmonary complications, wound infections, and systemic infectious complications were decreased [10].

The aims of the current study were to assess the results of open and laparoscopic splenectomy for nontraumatic splenic diseases performed at a single center in the laparoscopic era beyond the learning curve, and to identify patients who will benefit from either technique.

\section{Patients and methods}

\section{Patients}

A consecutive series of 52 patients undergoing elective OS or LS for various nontraumatic diseases at the Department of Visceral and Transplantation Surgery, University Hospital Zürich, from January 2001 until December 2006 were assessed retrospectively. Clinical data of all patients, including age, gender, ASA classification, indication for surgery, type of operation, intraoperative findings, operative complications, conversion rate, blood loss, operating time, postoperative morbidity, mortality, length of hospital stay, and histologic and morphologic findings of the removed specimen, were recorded in a database. The preoperative spleen size was determined by varying techniques (ultrasound, CT scan, MRI). The spleen volume assessed in this study was calculated as length $\times$ width $\times$ depth from the pathologist's measurements. In case of spleens that were morcellated during LS, the pieces were reassembled by the pathologist and then measured. The size was rather underestimated in those specimens.

Complications were classified and graded according to a complication classification that was recently published by our group [11]. Grade I and Grade II complications contain minor deviations compared to a normal postoperative course that can be treated with drugs, blood transfusion, physiotherapy, and nutritional supply. Grade III complications are characterized by the need of interventional treatment. Grade IV complications are life-threatening complications with ICU management. Grade V means death of the patient.

\section{Operative techniques}

Laparoscopic splenectomy was performed as previously described [12, 13]. Briefly, patients were positioned in a right lateral decubitus position. The stomach was decompressed with a nasogastric tube, and an antibiotic singleshot of $1 \mathrm{~g}$ cefazoline (Kefzol ${ }^{\circledR}$, Cerner Multum, Denver, CO) was given intravenously. A $10-\mathrm{mm}$ Hasson port was inserted into the left medioclavicular line. Two 12-mm ports were inserted under visual control along the costal margin on the patient's left side. A $10-\mathrm{mm} 30^{\circ}$ angled telescope was used. Usually the dissection was performed using a 5-mm ultrasonic dissector. The spleen was mobilized starting at the lateroposterior side by dividing the splenocolic and splenodiaphragmatic attachments. Then the short gastric vessels were divided, and the splenic hilum was completely freed. The pancreas was carefully dissected from the splenic hilum. Splenic vessels were divided using a 45-mm vascular stapler. Clips were used to close additional segmental splenic vessels. Finally, the spleen was put into an endoscopic bag, morcellated by instrumental clamping or finger fraction, and retrieved through an enlarged 15-mm trocar incision. The abdominal cavity was carefully assessed for accessory spleens.

Open splenectomy was performed either through a midline laparotomy or left subcostal incision, depending on the individual surgeon's preference. Then the lesser sac was opened and the splenic artery was dissected at the upper border of the pancreas, either using an endoscopic vascular stapler or traditional ligatures. The spleen was then completely freed.

All patients received pneumococcal and hemophilus B vaccines either 2 weeks before or after surgery. Meningococcal vaccines were not routinely given. All specimens were assessed for histologic investigation.

Statistical analysis

Results were expressed as median and range values. Groups were compared using Fisher's exact test and the Mann-Whitney test, where applicable. A $p<0.05$ was considered statistically significant. All analyses were performed using the SPSS v13.0 (SPSS, Inc., Chicago, IL).

\section{Results}

LS was performed in 25 patients, while 27 patients underwent OS. Patients who had LS were significantly 
younger $\quad(\mathrm{LS}, \quad$ median age $=41$ years, range $=24$ 65 years; OS, median age $=60$ years, range $=24$ 86 years; $p<0.001$ ), and had a significantly lower ASA score (ASA I: 1 patient, ASA II: 17 patients, ASA III: 6 patients, unknown: 1 patient) compared to OS patients (ASA II: 11 patients, ASA III: 13 patients, ASA IV: 1 patient, undetermined: 2 patients) ( $p=0.013)$. Preexisting cardiovascular disease was similar (LS, 5 patients; OS, 8 patients, $p=0.37$ ). The main underlying disease in the LS group was autoimmune thrombocytopenia in 18 patients, whereas OS was performed mostly for lymphoma (10 patients) or osteomyelofibrosis (7 patients). Three patients underwent LS and four patients underwent OS for hair cell leukemia. One patient underwent LS and three patients underwent OS for infectious reasons (Table 1). Two patients in the LS group and four patients in the OS group had had previous abdominal surgery.

The operation time was significantly longer for OS (median $=150 \mathrm{~min}$, range $=70-260 \mathrm{~min}$ ) than for LS $($ median $=107.5 \mathrm{~min}$, range $=50-390 \mathrm{~min}, p=0.028)$.

Conversion from LS to OS was necessary in two patients $(8 \%)$. One patient had a massively enlarged spleen that could not be handled laparoscopically, and the other patient required conversion because of intraoperative bleeding.

Intraoperative blood loss was significantly higher in OS patients (OS, median blood loss $=400 \mathrm{ml}$, range $=50$

Table 1 Patient charactistics

\begin{tabular}{|c|c|c|c|}
\hline & LS & OS & $p$ value \\
\hline Median age (years) & $41(24-65)$ & $60(24-86)$ & $<0.001$ \\
\hline Male/female & $13 / 12$ & $18 / 9$ & ns \\
\hline ASA II/III & $17 / 6$ & $11 / 13$ & 0.013 \\
\hline \multicolumn{4}{|l|}{ Splenic disease } \\
\hline ITP & 11 & 0 & \\
\hline TTP & 3 & 0 & \\
\hline Hair cell leukemia & 3 & 4 & \\
\hline Myeloproliferative syndrome & 0 & 8 & \\
\hline Non-Hodgkin's lymphoma & 0 & 5 & \\
\hline Chronic lymphatic leukemia & 0 & 2 & \\
\hline Other lymphoma & 2 & 2 & \\
\hline Splenic abscesses & 0 & 3 & \\
\hline Unclear splenomegaly & 0 & 2 & \\
\hline Hodgkin's lymphoma & 0 & 2 & \\
\hline Spherocytosis & 1 & 0 & \\
\hline Autoimmune hemolytic anemia & 3 & 0 & \\
\hline Necrobiotic xantogranuloma & 1 & 0 & \\
\hline Unclear infection & 1 & 0 & \\
\hline Cystic spleenic lymphangioma & 0 & 1 & \\
\hline Splenomegaly and HIV & 0 & 1 & \\
\hline
\end{tabular}

OS = open splenectomy; LS = laparoscopic splenectomy
$1500 \mathrm{ml} ; \mathrm{LS}$, median blood loss $=100 \mathrm{ml}$, range $=10$ $1000 \mathrm{ml} ; p=0.001)$. Intraoperative red blood cell substitution was given in seven OS patients and in one LS patient $(p=0.014)$. Platelet substitution was needed in three OS patients. Preoperative thrombocytopenia less than $80,000 / \mu 1$ was present in significantly more LS patients (LS, 13 patients, range $=13,000-67,000 / \mu \mathrm{l} ;$ OS, 9 patients, range $=$ $11,000-71,000 / \mu \mathrm{l} ; p<0.05)$.

Additional surgical procedures were performed in both groups. In the LS group, two patients underwent unplanned left pancreas resection because the pancreas tail was attached to the splenic hilum. In one patient, an accessory spleen was detected intraoperatively and removed. In the OS group, two left pancreas resections, one nephrectomy because of a papillary renal cell carcinoma, one cholecystectomy, one staging laparotomy because of Hodgkin's disease, and one abdominal wall reconstruction were performed. The operating time was longer in both groups compared to that of splenectomy alone (LS + additional procedure, median time $=125 \mathrm{~min}$, range $=105-140 \mathrm{~min}$; $\mathrm{OS}+$ additional procedure, median time $=150 \mathrm{~min}$, range $=120-180 \mathrm{~min}$ ) .

Spleen volume was significantly greater in OS patients (median volume $=2520 \mathrm{ml}$, range $=150-16,800 \mathrm{ml}$ ) than in LS patients (median volume $=684 \mathrm{ml}$, range $=150$ $4860 \mathrm{ml})(p=0.001)$.

Length of hospital stay was not different for OS (median $=8.5$ days, range $=3-26$ days) and LS (median $=$ 5 days, range $=2-146$ days). The patient who stayed in hospital for 146 days following LS received continued treatment at a medical unit for his underlying disease; the duration of his hospital stay was not related to the surgical procedure. Therefore, hospital stay of longer than 7 days was analyzed separately. Significantly more patients were hospitalized more than 7 days after OS (14 patients) than after LS (6 patients) $(p<0.05)$.

There was no 30-day mortality following LS; one patient died of multiorgan failure 26 days after OS. During follow-up, four more patients following OS died of sepsis.

Overall complication rate (Table 2) was lower in patients who underwent LS (LS, 8 patients, 23\%; OS, 13 patients, $48 \% ; p=0.027)$. Preoperative steroid treatment was significantly more frequent in patients who had LS (LS, 17 patients; OS, 9 patients; $p<0.05$ ), but no difference was observed in postoperative infectious complications. Postoperative pneumonia occurred in four patients following OS and was not observed after LS. Postoperative fever of unknown origin requiring antibiotic treatment developed in five patients in each group. One patient developed aortic valve endocarditis after OS and underwent successful emergency aortic valve replacement. Deep vein thrombosis (1 patient) and pulmonary embolism (1 patient) were observed only after LS. Pancreatic fistula developed in three 
Table 2 Mortality and complications following OS and LS

\begin{tabular}{llcc}
\hline & LS & OS & $p$ value \\
\hline Overall mortality & 0 & 5 & 0.052 \\
$\quad$ within 30 days & 0 & 1 & \\
Overall complications & 8 & 13 & 0.027 \\
$\quad$ Infection & 5 & 5 & \\
Pneumonia & 0 & 4 & \\
Rebleeding & 0 & 4 & \\
Pancreatic fistula & 3 & 0 & \\
Thromboembolic events & 2 & 0 & \\
\hline
\end{tabular}

OS = open splenectomy; LS = laparoscopic splenectomy

patients following LS; two patients underwent percutaneous drainage and one patient was treated conservatively. None of these three patients had undergone additional left lateral pancreatic resection intraoperatively. No pancreatic fistula was observed following OS. Reintervention because of bleeding was not necessary following LS, but it occurred in four patients following OS. In one of these patients, superficial wound revision revealed the source of bleeding; the other three patients required relaparotomy. Postoperative portal vein thrombosis was not observed.

None of the patients who underwent LS experienced higher-graded complications (Table 3) which required intervention under anesthesia (Grade IIIb and above). All complications following LS were graded I (3), II (3), and IIIa (2). Following OS, no grade I complications occurred, but higher-graded complications were more frequent (II: 4, IIIa: 0, IIIb: 3, IVa: 0, IVb: 1, V: 5).

No late complications were observed in either group, and no intervention for incisional hernia was necessary.

Table 3 Severity of surgical complications following OS and LS

\begin{tabular}{llll}
\hline & LS & OS & $p$ value \\
\hline Grade I & 3 & 0 & 0.026 \\
Grade II & 3 & 4 & \\
Grade IIIa & 2 & 0 & \\
Grade IIIb & 0 & 3 & \\
Grade Iva & 0 & 0 & \\
Grade Ivb & 0 & 1 & \\
Grade V & 0 & 5 & \\
\hline
\end{tabular}

Grade I: any deviation from the normal postoperative course without the need for pharmacologic or surgical intervention. Grade II: requiring pharmacologic treatment or blood transfusions. Grade III: requiring surgical endoscopic or radiologic intervention (IIIa) under local anesthesia or (IIIb) under general anesthesia. Grade IV: lifethreatening complication including, (IVa) single organ failure and (IVb) multiorgan failure. Grade V: death. OS = open splenectomy; LS = laparoscopic splenectomy

\section{Discussion}

In the present study, the results of a consecutive series of 52 patients who underwent elective total splenectomy for various diseases of the spleen were assessed. The indication for elective splenectomy is given by the underlying disease, whereas the technical approach is mainly determined by the size and weight of the spleen. The technical approach (open or laparoscopic) was chosen by the individual surgeon. Therefore, this study reflects the day-byday reality of the technical choice.

In general, severe splenomegaly is thought to interfere with the laparoscopic approach $[6,14]$. It has been suggested that laparoscopic splenectomy be used only in patients with a spleen weight lower than $1 \mathrm{~kg}$, since increased morbidity and a higher conversion rate were previously reported for patients with larger spleens [5, 14]. However, it was demonstrated recently that massively enlarged spleens of a craniocaudal length greater than $22 \mathrm{~cm}$ could be safely removed laparoscopically [9]. Different pathologies determine the size of the spleen, and a direct outcome comparison of the morbidity of patients who underwent OS or LS for the same underlying disease is missing in the literature. Therefore, the increased morbidity may not be related to the technique chosen but to the underlying disease. In this study, 9 of the 25 spleens (36\%) removed laparoscopically reached a volume of larger than $1000 \mathrm{ml}$. This points out that the previously suggested limit of $1 \mathrm{~kg}$ for laparoscopic surgery can be ignored and should not be an absolute reason to abstain from a laparoscopic procedure. Three of our patients had a spleen larger than $3000 \mathrm{ml}$. In one of these cases, a spleen of $4200 \mathrm{ml}$ was the reason for conversion; the other two were safely removed laparoscopically.

There is no prospective randomized trial comparing OS with LS, but several studies have shown the benefits of minimally invasive surgery. Less pain, decreased surgical morbidity, better cosmetic results, and a shortened hospital stay have been attributed to LS [15]. Other reports demonstrated only little $[9,16]$ or no shortening of the hospital stay following laparoscopic intra-abdominal surgery $[17,18]$. In this series, the median hospital stay was similar in both groups, but following LS, significantly more patients left the hospital after less than 7 days. The analysis of hospitalization time was complicated by the fact that some patients were referred to a medical unit following surgery to receive continued treatment for the underlying disease. Therefore, the time of hospitalization was overestimated in patients suffering from hematologic disorders since postoperative hospital stay was not determined only by the surgical procedure. This could also apply to previously published studies although it was not mentioned before. 
Patients who underwent LS in this study were about 20 years younger than those who had OS. It was previously demonstrated that patients older than 65 years had a higher overall complication rate after LS, but when stratified for ASA scores, the complication rates of OS and LS were similar [19]. This indicates that the patient's biological age alone should not guide the decision for laparoscopic or open splenectomy. In this study, OS was clearly preferred in patients with an ASA III score. However, OS was done mainly for malignant diseases in elderly patients with a higher ASA scores.

In contrast to previous reports [8, 10, 20], the median operation time in this series was significantly shorter for LS than for OS. This can be explained by several facts: LS was performed only by experienced surgeons, whereas OS was occasionally performed by younger surgeons as a teaching operation. Alternatively, this may reflect a conservative tendency of the surgeon: the open procedure was chosen a priori in cases that were judged as difficult preoperatively and in older patients with higher ASA scores. When there were additional procedures, either independent of the splenectomy or to treat complications, the median operation time was longer for both techniques, but again shorter in the laparoscopic group. Therefore, OS did not show an advantage with respect to complications.

Intraoperative bleeding during LS was a rare event and led to conversion to OS in only one case. Estimated blood loss was significantly lower in LS compared to OS. Portal hypertension and the degree of liver cirrhosis, which were previously identified as risk factors for high intraoperative blood loss during LS [21], were not present in any of the patients who had LS. In comparison, one patient in the OS group had portal vein thrombosis, but blood loss was moderate $(400 \mathrm{ml})$ and transfusion was not necessary.

The conversion rate of $8 \%$ observed in this study was lower than reported in similar studies [5, 8]. Conversion was not related to the learning curve of the laparoscopic procedure since the two conversion cases occurred in the middle (year 2002) and the end (year 2006) of the observation period. It had been demonstrated before that the conversion rate in LS was related to the institution's experience with this procedure [9]. Again, our low conversion rate reflects a conservative attitude of the surgeon, and open procedures were more likely to be chosen at the outset. The data of this study indicate that at the beginning of the observation period (years 2001 and 2002), OS was chosen for ten patients, and only four patients had LS. At the end of the observation period (years 2005 and 2006), LS was performed in 11 patients, and OS was done in only 6 patients. This demonstrates a trend to more confidence in the laparoscopic approach.

The overall complication rate of $33 \%$ was similar in both groups [10]. Winslow and Brunt [10] found in their meta-analysis an overall complication rate of $15 \%$ following LS. Complications in this study were mostly due to pancreatic fistula ( 3 patients) and thromboembolic events (2 patients), which did not occur after OS. Patients after OS experienced pneumonia (4 patients) and bleeding (3 patients).

Low platelet count was reported to be associated with higher morbidity [22]. Although in this study more patients who underwent LS presented with thrombocytopenia, the complication rate, including bleeding, was lower than in patients who had OS. This demonstrates that LS is also safe in the presence of a low platelet count. In line with other studies [23], the need for erythrocyte and platelet substitution was significantly lower in LS in this study, even though significantly more patients who had LS had severe thrombocytopenia before surgery. Because of the different origins of thrombocytopenia in LS patients (mainly immunothrombopenia) and in OS patients (mainly osteomyelofibrosis), this subgroup was not separately compared.

Portal vein thrombosis, reported in up to $14 \%$ of patients following LS [24], was not observed in this series, but was assessed only if clinically suspected. It was reported previously that by routine postoperative helical CT scan, portal vein thrombosis was detected in $55 \%$ of patients after LS, but in only $19 \%$ of patients after OS [25]. The subclinical incidence of portal vein thrombosis in this study was possibly underdiagnosed.

In conclusion, LS was performed in younger patients with moderate splenomegaly. Perioperative steroid treatment did not increase the rate of infectious or wound complications. Preoperative thrombocytopenia did not complicate LS. The conversion rate to OS was low. There were no technique-related differences between the two procedures with respect to morbidity. LS should primarily be attempted in all patients, if judged technically feasible and if the general condition of the patient allows laparoscopy. The size of the spleen alone should not drive the decision to an open approach.

\section{References}

1. Katz SC, Pachter HL (2006) Indications for splenectomy. Am Surg 72(7):565-580

2. Casaccia M, Torelli P, Squarcia S et al (2006) Laparoscopic splenectomy for hematologic diseases: a preliminary analysis performed on the Italian Registry of Laparoscopic Surgery of the Spleen (IRLSS). Surg Endosc 20(8):1214-1220

3. Silecchia G, Raparelli L, Casella G et al (2005) Laparoscopic splenectomy for non-traumatic diseases. Minerva Chir 60(5): 363-374

4. Burch M, Misra M, Phillips EH (2005) Splenic malignancy: a minimally invasive approach. Cancer J V11(1):36-42

5. Mahon D, Rhodes M (2003) Laparoscopic splenectomy: size matters. Ann R Coll Surg Engl 85(4):248-251 
6. Uranues S, Alimoglu O (2005) Laparoscopic surgery of the spleen. Surg Clin North Am 85:75-90

7. Boddy AP, Mahon D, Rhodes M (2006) Does open surgery continue to have a role in elective splenectomy? Surg Endosc 20:1094-1098

8. Targarona EM, Espert JJ, Cerdán G et al (1999) Effect of spleen size on splenectomy outcome. A comparison of open and laparoscopic surgery. Surg Endosc 13(6):559-562

9. Grahn SW, Alvarez J, Kirkwood K (2006) Trends in laparoscopic splenectomy for massive splenomegaly. Arch Surg 141(8):755761

10. Winslow ER, Brunt LM (2003) Perioperative outcomes of laparoscopic versus open splenectomy: a meta-analysis with emphasis on complications. Surgery 134:647-655

11. Dindo D, Demartines N, Clavien PA (2004) Classification of surgical complications: a new proposal with evaluation in a cohort of 6336 patients and results of a survey. Ann Surg 240(2):214-215

12. Delaitre B, Maignien B (1991) Splenectomy by the laparoscopic approach: report of a case. Presse Med 20:2263

13. Corcione F, Esposito C, Cuccurullo D et al (2002) Technical standardization of laparoscopic splenectomy. Surg Endosc 16:972-974

14. Patel AG, Parker JE, Wallwork B et al (2003) Massive splenomegaly is associated with significant morbidity after laparoscopic splenectomy. Ann Surg 238(2):235-240

15. Velanovich V, Shurafa MS (2001) Clinical and quality of life outcomes of laparoscopic and open splenectomy for haematological diseases. Eur J Surg 167(1):23-28
16. Lunevicius R, Monevicius M (2005) Systematic review comparing laparoscopic and open repair for perforated peptic ulcer. Br J Surg 92(10):1195-1207

17. Basse DL, Jacobsen DL, Bardram L et al (2005) Functional recovery after open versus laparoscopic colonic resection: a randomized, blinded study. Ann Surg 241(3):416-423

18. Moberg AC, Berndsen P, Palmquist I et al (2005) Randomized clinical trial of laparoscopic versus open appendicectomy for confirmed appendicitis. Br J Surg 92(3):298-304

19. Kavic SM, Segan RD, Park AE (2005) Laparoscopic splenectomy in the elderly: a morbid procedure? Surg Endosc 19:1561-1564

20. Walsh RM, Brody F, Brown N (2004) Laparoscopic splenectomy for lymphoproliferative disease. Surg Endosc 18(2):272-275

21. Ohta M, Nishizaki T, Matsumoto T et al (2005) Analysis of risk factors for massive intraoperative bleeding during laparoscopic splenectomy. J Hepatobiliary Pancreat Surg 12(6):433-437

22. Keidar A, Feldman M, Szold A (2005) Analysis of outcome of laparoscopic splenectomy for idiopathic thrombocytopenic purpura by platelet count. Am J Hematol 80(2):95-100

23. Vecchio R, Cacciola E, Lipari G et al (2005) Laparoscopic splenectomy reduces the need for platelet transfusion in patients with idiopathic thrombocytopenic purpura. JSLS 9(4):415-418

24. Harris W, Marcaccio M (2005) Incidence of portal vein thrombosis after laparoscopic splenectomy. Can J Surg 48(5):352-354

25. Ikeda M, Sekimoto M, Takiguchi S et al (2005) High incidence of thrombosis of the portal veinous system after laparoscopic splenectomy: a prospective study with contrast-enhanced CT scan. Ann Surg 241(2):208-216 